Article

\title{
Carvacrol Loaded Solid Lipid Nanoparticles of Propylene Glycol Monopalmitate and Glyceryl Monostearate: Preparation, Characterization, and Synergistic Antimicrobial Activity
}

\author{
Junbo He ${ }^{1,2} \oplus$, Shuangshuang Huang ${ }^{1}$, Xiaotao Sun ${ }^{3}$, Lijuan Han ${ }^{1,2}$, Chao Chang ${ }^{1,2}$, \\ Weinong Zhang ${ }^{1,2, *}$ and Qixin Zhong ${ }^{4}$ \\ 1 Key Laboratory for Deep Processing of Major Grain and Oil, Ministry of Education, College of Food Science \\ \& Engineering, Wuhan Polytechnic University, Wuhan 430023, China \\ 2 Hubei Key Laboratory for Processing and Transformation of Agricultural Products, Wuhan Polytechnic \\ University, Wuhan 430023, China \\ 3 Beijing Key Lab of Flavor Chemistry, Beijing Technology and Business University, Beijing 100048, China \\ 4 Department of Food Science, The University of Tennessee, Knoxville, TN 37996, USA \\ * Correspondence: zhangweinong@163.com; Tel.: +86-027-8392-4790
}

Received: 23 July 2019; Accepted: 12 August 2019; Published: 14 August 2019

check for

\begin{abstract}
To develop solid lipid nanoparticles (SLNs) with stable lipid matrix structures for the delivery of bioactive compounds, a new class of SLNs was studied using propylene glycol monopalmitate (PGMP) and glyceryl monostearate (GMS) mixtures and carvacrol as a model lipophilic antimicrobial. Stable SLNs were fabricated at PGMP:GMS mass ratios of 2:1 and 1:1, and the carvacrol loading was up to $30 \%$ of lipids with $>98 \%$ encapsulation efficiency and absence of visual instability. Fluorescence spectra and release profiles indicated the carvacrol was successfully encapsulated and homogeneously distributed within the SLNs. SLNs fabricated with equal masses of PGMP and GMS had better stability of carvacrol during storage and higher sphericity than those with a ratio of 2:1 and were much more effective than free carvacrol against Escherichia coli O157:H7 and Staphylococcus aureus. These findings demonstrated the potential applications of the studied SLNs in delivering lipophilic bioactive compounds in food and other products.
\end{abstract}

Keywords: solid lipid nanoparticles; carvacrol; propylene glycol monopalmitate; synergistic antimicrobial activity

\section{Introduction}

Solid lipid nanoparticles (SLNs) are a class of colloidal particles composed of solid lipids at the application temperature and have become a group of fascinating delivery vehicles in food, cosmetic, and medical sciences owing to the good biocompatibility [1-3]. Encapsulation of bioactive compounds, particularly poorly water-soluble or unstable ones, in SLNs can improve water-solubility of these compounds, as well as stability against oxidative, UV, and thermal stresses [4]. However, low loading capacity and polymorphic transformation during storage are two major drawbacks of SLNs greatly limiting their application [5]. Especially, the polymorphism transformation of lipid matrixes in SLNs results from rearrangement of crystalline lattices to form thermodynamically more stable polymorph, e.g., from $\alpha$ - to $\beta$-form crystals, and the resultant increase in lipid molecular ordering causes the expulsion of initially incorporated bioactive compounds [6,7]. Maintaining the crystalline structure or slowing the polymorphic transition is needed to overcome the challenges of SLNs as delivery systems. We recently reported that the SLNs prepared with a diacylglycerol, 1-laurin-3-palmitin, 
had a higher loaded capacity and better stability than those of glyceryl monostearate (GMS) and glyceryl tripalmitate due to the stable, mostly $\beta$-form, crystalline polymorph of 1-laurin-3-palmitin [8]. Therefore, identifying solid food lipids forming stable crystalline structures is a new direction to study SLNs for food application.

Propylene glycol monoesters (PGMEs) are widely used as food emulsifiers for cake mixes, sponge cakes, whipped toppings, and bread [9]. Furthermore, the purified PGMEs have specific crystalline properties with the ability to stabilize the meta-stable $\alpha$-form crystals of distilled GMS even for a year, and this kinetic stability meets the shelf-life of many aerated products [9-11]. The kinetically stable $\alpha$-form crystals formed of the mixture of PGME and GMS, also referred to as "conjoined crystals", provide a rational lipid matrix to fabricate SLNs. However, there is currently no study on SLNs prepared with PGME and GMS mixtures.

Antimicrobials are one group of bioactive compounds that can utilize the characteristics of SLNs as delivery systems, such as the release kinetics controllable by lipid matrix structures. Carvacrol (5-isopropyl-2-methylphenol) is a major phenolic constituent of essential oils derived from thyme, marjoram, and oregano [12]. Carvacrol possesses broad biological activities, such as antioxidant [13,14], antimicrobial [14-16], and antitumor activities [17]. As generally recognized as safe (GRAS) food additive, carvacrol has been widely used as a flavoring or antimicrobial agent in the food industry. However, the utilization of carvacrol faces some challenges including its poor-water solubility, strong odor, and instability when exposed to air, light, or heat. Encapsulation of carvacrol to increase the water solubility or stability has been reported for systems such as chitosan-based nanoparticles [18], $\beta$-cyclodextrin inclusion complexes [19], nanoemulsions [20], and zein nanoparticles [21]. However, SLNs are rarely studied to encapsulate carvacrol to utilize advantages of SLNs as delivery systems.

The first objective of the present study was to investigate the mixture of propylene glycol monopalmitate (PGMP) and GMS as novel lipid matrixes for fabricating of SLNs and the properties of encapsulating carvacrol. The second objective was to study the antimicrobial activity of carvacrol-loaded SLNs (Car-SLNs). The Car-SLNs were characterized for dimension using dynamic light scattering (DLS), zeta-potential, fluorescence spectra, release profile, and morphology using transmission electron microscopy (TEM). The antimicrobial activities of Car-SLNs were evaluated against model Gram-negative bacterium Escherichia coli O157:H7 and Gram-positive bacterium Staphylococcus aureus.

\section{Materials and Methods}

\subsection{Materials}

1,2-Propylene glycol (>99\% pure), palmitoyl chloride ( $>97 \%$ pure), carvacrol (99\% pure), and Tween 80 (T80) were purchased from Aladdin Bio-Chem Technology Co., Ltd. (Shanghai, China). GMS (analytic reagent) was purchased form Shanpu Chemical Co., Ltd. (Shanghai, China). The bacterial strains of E. coli O157:H7 was obtained from the Culture Collection of the College of Food Science and Engineering at Wuhan Polytechnic University and S. aureus (CCTCC AB 91093) was purchased from China Center for Type Culture Collection (Wuhan, China). Lysogeny broth (LB) was prepared by dissolving $25 \mathrm{~g}$ of powder, purchased from Qingdao Hope Bio-Technology Co., Ltd. (Qingdao, China), in $1000 \mathrm{~mL}$ deionized water and autoclaving at $121^{\circ} \mathrm{C}$ for $15 \mathrm{~min}$. Double distilled water $\left(\mathrm{ddH}_{2} \mathrm{O}\right)$ was used in the preparation of all samples. All other chemicals and solvents were of analytical grade and were used as received.

\subsection{Synthesis of Propylene Glycol Monopalmitate}

PGMP was prepared by the reaction of propylene glycol and palmitoyl chloride in the presence of triethylamine as the base, which is different from the report by the direct esterification method [22]. Briefly, a mixture of 1,2-propylene glycol $(10 \mathrm{mmol})$ and triethylamine $(14 \mathrm{mmol})$ in dichloromethane $(30 \mathrm{~mL})$ was cooled to $0{ }^{\circ} \mathrm{C}$ in ice-water, and palmitoyl chloride $(12 \mathrm{mmol})$ dissolved in dichloromethane $(5 \mathrm{~mL})$ was added dropwise. After stirring at room temperature for $3 \mathrm{~h}$, the mixture was washed 
with water $(30 \mathrm{~mL})$ and then $0.1 \mathrm{M} \mathrm{NaOH}$ solution $(30 \mathrm{~mL})$. The resulting organic phase was dried by $\mathrm{MgSO}_{4}$ overnight, concentrated under reduced pressure to harvest the PGMP as white solids that had a melting point of $42-44{ }^{\circ} \mathrm{C}$ and a yield of $88 \%$. Spectroscopic properties of the obtained PGMP powder are studied in detail in the Supporting Information, however, these data were not reported in the published work [22].

\subsection{Preparation of SLN Dispersions}

The SLN dispersions were prepared by a microemulsion template method (Scheme 1) [8]. Briefly, $90 \mathrm{mg}$ of lipids containing the mixture of PGMP and GMS at a mass ratio from 1:0, 2:1, 1:1, 1:2, to $0: 1$ was melted at $70{ }^{\circ} \mathrm{C}$ under continuous stirring. The aqueous phase was prepared by adding T80 (Tween $80,270 \mathrm{mg})$ to $\mathrm{ddH}_{2} \mathrm{O}(2 \mathrm{~g})$ at $70{ }^{\circ} \mathrm{C}$. The mass ratio of lipid to $\mathrm{T} 80$ was fixed at 1:3. Then, the aqueous phase was added rapidly to the lipid phase at $70^{\circ} \mathrm{C}$, after which, ethanol $(0.5 \mathrm{~mL})$ was added dropwise to the pre-emulsion and stirred for $5 \mathrm{~min}$ to form a transparent microemulsion. The hot microemulsion was subsequently dispersed in 5 parts $(v / v)$ of cold water $\left(2-4^{\circ} \mathrm{C}\right)$ to solidify lipids. All samples were stored at $25^{\circ} \mathrm{C}$ for further analysis.

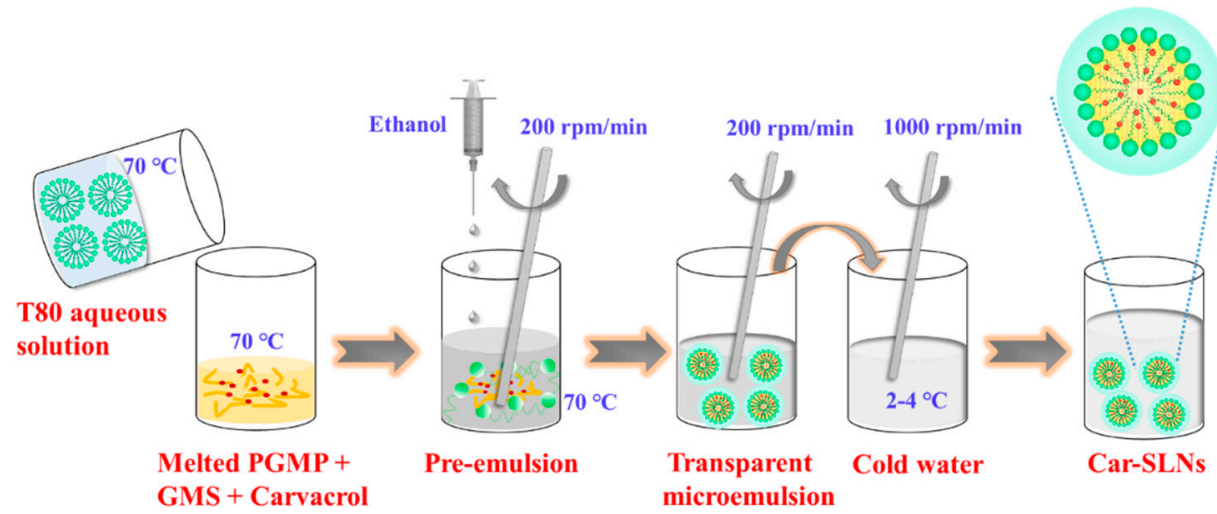

Scheme 1. The microemulsion method for the preparation of carvacrol-loaded solid lipid nanoparticles (Car-SLNs).

\subsection{Determination of Z-Average Mean Diameter, Polydispersity Index (PDI), and Zeta-Potential}

The Z-average mean diameter and PDI of the prepared SLN dispersions were determined using DLS (model Zetasizer Nano 90ZS, Malvern Instruments Ltd., Worcestershire, UK). The laser wavelength was set at $633 \mathrm{~nm}$, and the material/dispersant reflective index was 1.590/1.330. Samples were prepared by diluting the SLN dispersions 30-fold using $\mathrm{ddH}_{2} \mathrm{O}$. The zeta-potential was determined by microelectrophoresis on the same instrument.

\subsection{Preparation and Characterization of Carvacrol-Loaded SLNs}

To prepare Car-SLNs, carvacrol was dissolved at 20\%, 30\%, and 40\% mass of PGMP and GMS mixtures melted at $70{ }^{\circ} \mathrm{C}$, followed by preparing SLNs as above. Loading capacity of carvacrol was evaluated by adding different amounts of carvacrol into the lipids and preparing the SLNs dispersions based on the optimized formula. The Z-average mean diameter, PDI, and zeta-potential of prepared Car-SLNs after storage at $25^{\circ} \mathrm{C}$ for 0 and 30 days were determined as in the previous section.

\subsection{Determination of Entrapment Efficiency of Car-SLNs}

Entrapment efficiency (EE) was determined using an ultrafiltration method with some modification [23]. The Car-SLN dispersions were passed through a flat polyethersulfone (PES) ultrafiltration membrane (SEPRO Membranes, Inc., USA) with a molecular weight cur-off (MWCO) of $10 \mathrm{kDa}$ using an ultrafiltration cup (UHP-25K, Advantech Toyo, Tokyo, Japan) under constant nitrogen 
passage of $0.45 \mathrm{MPa}$. The free carvacrol in filtrate was analyzed with a HPLC method presented below. The EE was calculated using the following Equation (1):

$$
\mathrm{EE}(\%)=\left[\left(\mathrm{W}_{\mathrm{t}}-\mathrm{W}_{\mathrm{f}}\right) / \mathrm{W}_{\mathrm{t}}\right] \times 100 \% \text {, }
$$

where $\mathrm{W}_{\mathrm{t}}$ and $\mathrm{W}_{\mathrm{f}}$ are the total mass of carvacrol added and the mass of free carvacrol, respectively.

\subsection{Chemical Stability of Carvacrol Loaded in SLNs}

The chemical stability of carvacrol after storage at $25^{\circ} \mathrm{C}$ for seven and 30 days was evaluated to investigate the protection effect of SLNs. To quantify residual carvacrol, $200 \mu \mathrm{L}$ of a Car-SLN dispersion was dissolved in dry methanol $(2 \mathrm{~mL})$ and sonicated for $30 \mathrm{~min}$, and then $200 \mu \mathrm{L}$ of the mixture was diluted by 10 times using methanol and used for HPLC analysis. The Waters 1525 HPLC system (Waters Corporation, Milford, MA, USA) was equipped with a UV-vis detector at $274 \mathrm{~nm}$ and an Agilent C18 column $(250 \mathrm{~mm} \times 4.5 \mathrm{~mm}, 5 \mu \mathrm{m})$ kept at $30^{\circ} \mathrm{C}$. The mobile phase was composed of acetonitrile and deionized water $(60: 40, v / v)$ and run at a flow rate of $1 \mathrm{~mL} / \mathrm{min}$ during isocratic separation. The carvacrol content was determined by referring to a calibration curve established with standard solutions of carvacrol dissolved in methanol $\left(R^{2}=0.998\right)$. The chemical stability was determined by calculating the percentage of residual carvacrol with reference to that on day 0 .

\subsection{Fluorescence Study of Car-SLN Dispersions}

The interaction between carvacrol and SLNs was studied with fluorescence spectrophotometry (model F-4600, Hitachi, Ltd., Tokyo, Japan). Car-SLN dispersions were diluted by nine times with $\mathrm{ddH}_{2} \mathrm{O}$ to fit the instrument sensitivity and the fluorescence spectra were obtained at an excitation wavelength of $227 \mathrm{~nm}$ and an emission wavelength range from 200 to $500 \mathrm{~nm}$. The slit width was $5 \mathrm{~nm}$ for both excitation and emission. Carvacrol pre-dissolved in ethanol and $\mathrm{ddH}_{2} \mathrm{O}(0.3 \mathrm{mg} / \mathrm{mL})$, and SLN dispersions without carvacrol were studied as controls.

\subsection{Release Kinetics of Carvacrol from the SLNs}

The release kinetics of carvacrol in SLNs was evaluated using a dialysis membrane method [24]. Briefly, the freshly prepared Car-SLN dispersion was diluted with release medium (water/ethanol, 9:1 $v / v$ ) to a carvacrol amount of $2.0 \mathrm{mg}$. The membrane bag (molecular weight cut-off of $3500 \mathrm{Da}$ ) filled with $10 \mathrm{~mL}$ of the diluted Car-SLN dispersion was immersed in $400 \mathrm{~mL}$ of the release medium that was stirred at $100 \mathrm{rpm}$ and $25^{\circ} \mathrm{C}$. Two milliliter aliquots of the release medium were withdrawn at predetermined time intervals and the same amount of the release medium was replenished. The carvacrol in the collected samples was determined using the above HPLC protocol. The release kinetics of free carvacrol was performed in the same manner for $10 \mathrm{~mL}$ of $2.0 \mathrm{mg}$ carvacrol dissolved in ethanol. All measurements were performed in three replicates.

\subsection{Transmission Electron Microscopy}

The morphology of SLNs with the carvacrol loading of 30\% in the lipid phase after 30 days storage at $25{ }^{\circ} \mathrm{C}$ was evaluated using a Hitachi HT7700 transmission electron microscope (Hitachi High-Technologies, Pleasanton, CA, USA). The Car-SLN dispersion was initially deposited onto a 200-mesh copper grid and allowed to dry for $15 \mathrm{~min}$. After drying, the sample negatively stained with $2 \%$ phosphotungstic acid was analyzed at a voltage of $80 \mathrm{kV}$.

\subsection{Antimicrobial Activity of Car-SLNs}

The minimum inhibitory concentration (MIC) and minimum bactericidal concentration (MBC) of free carvacrol, bare SLNs, and Car-SLNs with 30\% loading against both Gram-negative E. coli O157:H7 and Gram-positive S. aureus were determined by the microbroth dilution method according to a previous study with slight modification [25]. Free carvacrol stock solution was prepared by 
diluting the carvacrol solution $(2 \mathrm{mg} / \mathrm{mL}$ in ethanol) to $1 \mathrm{mg} / \mathrm{mL}$ with $\mathrm{LB}$. From the stock solution, the working solutions were prepared by further diluting the stock solution using LB to 0.1, 0.2, 0.25, 0.3, $0.4,0.5,0.6,0.7,0.8$, and $1 \mathrm{mg} / \mathrm{mL}$ of carvacrol. Car-SLNs dispersions were directly diluted to the same concentrations of free carvacrol with LB. To wells of sterile 96-well microtiter plates were first added $120 \mu \mathrm{L}$ of bacterial culture (ca. $10^{6} \mathrm{CFU} / \mathrm{mL}$ bacteria) followed by $120 \mu \mathrm{L}$ of the antimicrobial sample. Absorbance of wells at $630 \mathrm{~nm}$ was measured using an automated microplate reader (PerkinElmer EnSpire, Waltham, MA, USA) before and after $24 \mathrm{~h}$ incubation at $37^{\circ} \mathrm{C}$. The MIC was defined as the carvacrol concentration that allowed less than or equal to a 0.05 increase in absorbance after $24 \mathrm{~h}$ incubation. To determine $\mathrm{MBC}, 20 \mu \mathrm{L}$ of the mixture from wells showing no growth was spread on LB plates. Following incubation of LB plates at $37^{\circ} \mathrm{C}$ for $24 \mathrm{~h}$, the lowest carvacrol concentration corresponding to no bacterial growth was defined as the MBC. The bare SLN dispersions were used as a control and diluted as the Car-SLNs in the assay.

\subsection{Statistical Analysis}

All experiments were performed in triplicate. The statistical analyses were conducted using SPSS software (IBM SPSS statistic 19) with one-way ANOVA and Tukey's multiple comparison test to determine the difference among samples. The significant level $(p)$ was set as 0.05 .

\section{Results and Discussion}

\subsection{Synthesis of PGMP}

To form kinetically stable $\alpha$-form crystals, the purity of PGMEs needs to be $90 \%$ or higher $[10,11]$. The purity of PGMP synthesized in the present study was evaluated using ${ }^{1} \mathrm{H}$ NMR, and no propylene glycol dipalmitate was detected (Supplementary Figure S1). The high purity of PGMP can be attributed to the steric effect that the formation of PGMP hinders the further esterification with another palmitoyl chloride molecule. The FT-IR spectra of PGMP (Supplementary Figure S2) presented in the supporting information revealed typical characteristic peaks, such as the broad peak at $3404 \mathrm{~cm}^{-1}$ (stretching vibration of $\mathrm{OH}$ ), sharp peak at $1743 \mathrm{~cm}^{-1}$ (stretching vibration of esterified carbonyl group), sharp peak at $1460 \mathrm{~cm}^{-1}$ (bending vibration of methylene group), and sharp peak at $725 \mathrm{~cm}^{-1}$ (rocking vibration of long methylene chain). The mass spectrum of PGMP (Supplementary Figure S3) was observed at the $m / z$ of $337.37\left([\mathrm{M}+\mathrm{Na}]^{+}\right)$. Therefore, the synthesized PGMP had a sufficiently high purity to prepare SLNs.

\subsection{Effect of the Mass Ratio of PGMP and GMS on SLN Formation}

To construct stable SLNs dispersions, the mass ratio of PGMP and GMS was optimized firstly using T80 as a surfactant at the one-third mass of solid lipids (PGMP + GMS). The Z-average mean diameter, PDI, zeta-potential, and visual stability of SLN dispersions prepared by using different PGMP:GMS mass ratios are summarized in Table 1. With the increase of the PGMP:GMS mass ratio from 1:0 to 1:1, the Z-average mean diameter, PDI, and zeta-potential magnitude of these SLNs dispersions all consistently increased. The PDI was all smaller than 0.3 , indicating relatively monodispersed SLNs. The impact of lipid composition on Z-average mean diameter can be attributed to the polarity and melting point/viscosity of lipids during microemulsion formation. PGMP has a hydrophile-lipophile balance (HLB) value (4.6) higher than that of GMS (3.6) calculated according to Equation (2). An HLB value of the lipid phase closer to that of surfactant (15.0 for T80) lowers the interfacial tension and facilitates formation of smaller lipid droplets in microemulsions [26]. An increase in GMS content lowers the HLB of lipid mixture to be further away from that of T80 and therefore is expected to increase the dimension of SLNs, which agrees with the data in Table 1. A higher melting point/viscosity 
of GMS $\left(58-60^{\circ} \mathrm{C}\right)$ than PGMP $\left(42-44^{\circ} \mathrm{C}\right)$ [27] increases the interfacial tension and contributes to the increase in droplet size of microemulsions.

$$
\text { HLB }=20 \times\left[M_{\text {Hydrophilic }} /\left(M_{\text {Hydrophilic }}+M_{\text {Lipophilic }}\right)\right],
$$

where $\mathrm{M}_{\text {Hydrophilic }}$ and $\mathrm{M}_{\text {Lipophilic }}$ are the molecular mass of hydrophilic and lipophilic (palmitate or stearate) portions of a surfactant [28].

Table 1. Effects of mass ratio of PGMP and GMS on the Z-average mean diameter (Z-average), polydispersity index (PDI), zeta-potential, and visual stability of solid lipid nanoparticle dispersions.

\begin{tabular}{ccccc}
\hline PGMP:GMS & Z-Average $\mathbf{( n m )}$ & PDI & Zeta-Potential $(\mathbf{m V})$ & Visual Stability \\
\hline $1: 0$ & $14.9 \pm 0.4$ & $0.133 \pm 0.017$ & $5.6 \pm 0.9$ & Precipitation after 3 days \\
$2: 1$ & $18.6 \pm 0.2$ & $0.178 \pm 0.008$ & $-11.0 \pm 0.1$ & Stable for 1 month \\
$1: 1$ & $25.3 \pm 0.7$ & $0.296 \pm 0.003$ & $-13.7 \pm 0.8$ & Stable for 1 month \\
$1: 2$ & ND & ND & ND & Precipitation after 1 day \\
$0: 1$ & ND & ND & ND & Precipitation after 1 day \\
\hline
\end{tabular}

${ }^{1} \mathrm{ND}$ : Not determined due to precipitation.

Visual stability of SLNs is also summarized in Table 1. SLNs prepared with PGMP:GMS mass ratios of 2:1 and 1:1 were still stable after one month, whereas those with PGMP only precipitated after three days and those with PGMP:GMS mass ratios of 1:2 and 0:1 showed precipitation after one day. PGMP:GMS mass ratios of 2:1 and 1:1 were chosen to prepare Car-SLNs.

\subsection{Properties of SLNs Encapsulating Carvacrol}

On the basis of the optimized formulations for bare SLNs dispersions, carvacrol was encapsulated in the SLNs with PGMP:GMS mass ratios of 2:1 and 1:1. Effects of loading capacities of carvacrol on the Z-average mean diameter, PDI, zeta-potential, and EE were investigated and the results are shown in Table 2. The EEs were all higher than $98 \%$. The Z-average mean diameters of freshly prepared Car-SNLs using the PGMP:GMS mass ratio of 2:1 were smaller than those with the PGMP:GMS mass ratio of 1:1, showing the same trend as the bare SLNs (Table 1). The significant growth of the nanoparticle dimension was observed after 30-day storage, more significant at a PGMP:GMS mass ratio of 2:1 than at 1:1, suggesting particle aggregation. After storage for 30 days, SLNs with a PGMP:GMS mass ratio of 2:1 showed the appearance of smaller particles, in addition to shifting to bigger particles (Figure 1a), which is the characteristics of Ostwald ripening [29]. Physically, as carvacrol has a solubility of 0.45 $\mathrm{mg} / \mathrm{mL}$ in water and $1.63 \mathrm{mg} / \mathrm{mL}$ in $5 \%$ aqueous ethanol and carvacrol in smaller particles has a higher solubility $[29,30]$, carvacrol in smaller particles will dissolve into the continuous phase, and the dissolved carvacrol will join in bigger droplets to lower system free energy. As a result, smaller particles become smaller, while bigger particles become bigger after storage. In contract, SLNs prepared with a PGMP:GMS mass ratio of 1:1 showed the shifting to bigger particles and an appearance of big particles of one order of magnitude higher (Figure 1b), which may indicate particle aggregation. As the zeta-potential magnitude of SLNs is smaller than 20 (Table 2), the electrostatic repulsion may not be strong enough to prevent particle aggregation [31]. The absence of Ostwald ripening for SLNs at a PGMP:GMS ratio of 1:1 may result from the higher melting point (mass transfer resistance for carvacrol) of the lipid matrix than SLNs at a PGMP:GMS ratio of 2:1, as discussed previously. The aggregation and/or Ostwald ripening resulted in the widened particle size distribution (Figure 1) and increased PDI (Table 2) after storage. Nevertheless, the z-average mean diameter of all treatment was smaller than $200 \mathrm{~nm}$ after storage and all SLNs remained visually stable, except for minor precipitation observed at the highest carvacrol level treatments. 
Table 2. The Z-average mean diameter (Z-average), polydispersity index (PDI), zeta-potential, and encapsulation efficiency (EE\%) values for the Car-SLNs dispersions ${ }^{1}$.

\begin{tabular}{|c|c|c|c|c|c|c|c|c|}
\hline \multirow{2}{*}{ PGMP:GMS } & \multirow{2}{*}{$\begin{array}{l}\text { Carvacrol Loading } \\
\text { (\% Mass of Lipids) }\end{array}$} & \multicolumn{2}{|c|}{ Z-Average (nm) } & \multicolumn{2}{|c|}{ PDI } & \multicolumn{2}{|c|}{ Zeta-Potential (mV) } & \multirow{2}{*}{$\begin{array}{l}\text { EE (\%) } \\
\text { 0 Days }\end{array}$} \\
\hline & & 0 Day & 30 Days & 0 Day & 30 Days & 0 Day & 30 Days & \\
\hline \multirow{4}{*}{$2: 1$} & 0 & $18.6 \pm 0.2^{d}$ & $32.9 \pm 0.5^{\mathrm{d}}$ & $0.178 \pm 0.008^{a}$ & $0.292 \pm 0.027^{b}$ & $-11.0 \pm 0.1^{\mathrm{ab}}$ & $-14.1 \pm 1.6^{c}$ & \\
\hline & 20 & $39.8 \pm 0.6^{c}$ & $149.4 \pm 1.8^{c}$ & $0.184 \pm 0.010^{a}$ & $0.221 \pm 0.018^{c}$ & $-9.73 \pm 0.1^{b}$ & $-18.5 \pm 0.7^{b}$ & $98.0 \pm 0.1^{c}$ \\
\hline & 30 & $68.9 \pm 0.1^{b}$ & $159.8 \pm 2.5^{b}$ & $0.182 \pm 0.012^{a}$ & $0.313 \pm 0.002^{a, b}$ & $-12.6 \pm 1.7^{\mathrm{a}}$ & $-21.2 \pm 1.6^{a, b}$ & $98.3 \pm 0.1^{\mathrm{a}}$ \\
\hline & 40 & $95.4 \pm 0.7^{\mathrm{a}}$ & $165.8 \pm 3.4^{\mathrm{a}}$ & $0.182 \pm 0.008^{a}$ & $0.355 \pm 0.051^{\mathrm{a}}$ & $-11.9 \pm 1.4^{\mathrm{a}, \mathrm{b}}$ & $-23.2 \pm 2.9^{\mathrm{a}}$ & $98.2 \pm 0.2^{\mathrm{a}, \mathrm{b}}$ \\
\hline \multirow{4}{*}{$1: 1$} & 0 & $25.3 \pm 0.7^{\mathrm{d}}$ & $55.1 \pm 0.2^{d}$ & $0.296 \pm 0.003^{a}$ & $0.278 \pm 0.003^{a}$ & $-13.7 \pm 0.8^{b}$ & $-15.3 \pm 1.3^{c}$ & \\
\hline & 20 & $56.1 \pm 0.3^{c}$ & $98.7 \pm 0.9^{c}$ & $0.211 \pm 0.010^{c}$ & $0.198 \pm 0.002^{c}$ & $-17.1 \pm 0.6^{\mathrm{a}}$ & $-17.4 \pm 1.1^{b}$ & $99.2 \pm 0.1^{b}$ \\
\hline & 30 & $89.3 \pm 1.2^{b}$ & $155.2 \pm 2.7^{b}$ & $0.224 \pm 0.008^{c}$ & $0.233 \pm 0.004^{b}$ & $-17.9 \pm 1.4^{a}$ & $-18.6 \pm 1.2^{\mathrm{a}, \mathrm{b}}$ & $99.4 \pm 0.1^{\mathrm{a}}$ \\
\hline & 40 & $115.8 \pm 0.8^{a}$ & $193.5 \pm 2.5^{\mathrm{a}}$ & $0.239 \pm 0.005^{b}$ & $0.228 \pm 0.016^{\mathrm{b}}$ & $-16.6 \pm 0.6^{a}$ & $-19.9 \pm 0.5^{a}$ & $98.1 \pm 0.1^{\mathrm{c}}$ \\
\hline
\end{tabular}

${ }^{1}$ Data not sharing the same superscript letter in the same column are significantly different $(p<0.05)$. 


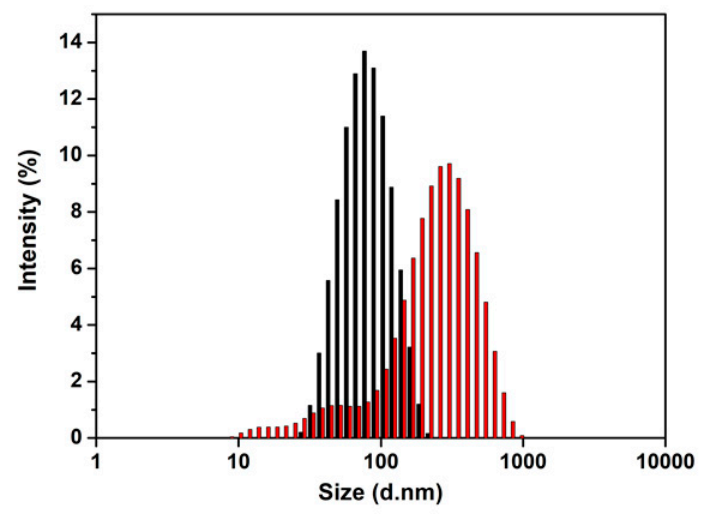

(a)

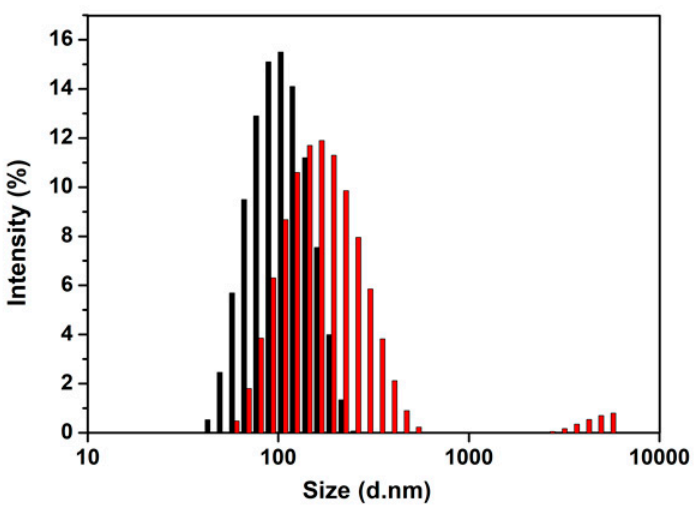

(b)

Figure 1. Intensity size distributions of SLNs prepared with a PGMP:GMS mass ratio of 2:1 (a) or 1:1 (b) and a carvacrol loading at 30\% mass of lipids, before (black column) and after (red column) 30-day storage at $25^{\circ} \mathrm{C}$.

\subsection{Chemical Stability of Carvacrol in SLNs}

Carvacrol as a natural bioactive compound is chemically unstable since it is sensitive to light and oxygen [32]. Therefore, the chemical stability of carvacrol was evaluated over 30 days. The results are shown in Table 3. Free carvacrol dissolved in ethanol to a concentration equivalent to the Car-SLNs with a carvacrol loading at $30 \%$ mass of lipids showed the reduction to $84.1 \%$ and $80.0 \%$ after seven and 30 days of storage, respectively. Nanoencapsulation of carvacrol in SLNs improved the chemical stability. Car-SLNs fabricated with a PGMP:GMS mass ratio of 1:1 displayed a better stability ( $>95 \%$ residual carvacrol) than those of a PGMP:GMS mass ratio of 2:1 ( 92\%) after seven-day storage. The same trend was observed on day 30, and the higher residual carvacrol\% in Car-SLNs was observed at a lower carvacrol loading. As minor precipitation was observed for SLNs prepared with a carvacrol loading at $40 \%$ mass of lipids, the optimum carvacrol loading capacity at the studied conditions was concluded for $30 \%$ of the lipids, which was used in subsequent studies.

Table 3. Chemical stability of free carvacrol and carvacrol loaded in SLNs.

\begin{tabular}{|c|c|c|c|}
\hline \multirow{2}{*}{ PGMP:GMS } & \multirow{2}{*}{ Carvacrol Loading (\% Mass of Lipids) } & \multicolumn{2}{|c|}{ Residual Carvacrol (\%) ${ }^{2}$} \\
\hline & & 7 day & 30 days \\
\hline \multirow{3}{*}{$2: 1$} & 20 & $91.6 \pm 3.9^{b}$ & $90.3 \pm 3.1^{\mathrm{ab}}$ \\
\hline & 30 & $92.7 \pm 2.1 \mathrm{ab}$ & $86.5 \pm 2.2^{b c}$ \\
\hline & 40 & $91.6 \pm 3.0^{b}$ & $83.7 \pm 3.9 \mathrm{~cd}$ \\
\hline \multirow{3}{*}{$1: 1$} & 20 & $95.7 \pm 3.1^{\mathrm{a}}$ & $92.0 \pm 2.8^{\mathrm{a}}$ \\
\hline & 30 & $95.9 \pm 0.7^{\mathrm{a}}$ & $92.6 \pm 1.3^{a}$ \\
\hline & 40 & $95.0 \pm 2.0^{\mathrm{a}}$ & $87.1 \pm 1.2^{b c}$ \\
\hline Free carvacrol ${ }^{1}$ & & $84.1 \pm 1.1^{\mathrm{c}}$ & $80.0 \pm 1.8^{\mathrm{d}}$ \\
\hline
\end{tabular}

${ }^{1}$ Free carvacrol was prepared in ethanol at the same concentration as SLNs with a carvacrol loading at $30 \%$ mass of lipids. ${ }^{2}$ Data not sharing the same superscript letter in the same column are significantly different $(p<0.05)$.

The chemical stability of carvacrol in SLNs may be related to the crystal stability. Previous studies indicated that an equal molar ratio of PGME and GMS was the most effective mixture for retaining $\alpha$-form crystals, while other molar ratios resulted in the polymorphic transformation to the more stable high-melting $\beta$-form crystals [10]. In this study, SLNs with equal masses of PGMP (MW = $314 \mathrm{Da}$ ) and GMS (MW = $358 \mathrm{Da}$ ) had a molar ratio of almost 1:1. Therefore, the SLNs formulated with a PGMP:GMS mass ratio of 1:1 displayed better carvacrol stability during storage, possibly attributed to the less expulsion of carvacrol due to the stable $\alpha$-form crystals than those with a PGMP:GMS mass ratio of 2:1. 


\subsection{Interactions between Lipids and Carvacrol Studied with Fluorescence Spectroscopy}

Fluorescence spectroscopy was used to investigate the interaction of carvacrol with SLNs, with comparison to the respective bare SLN dispersions and free carvacrol. Figure 2 shows strong fluorescence intensity of carvacrol dissolved in ethanol, with the maximum at a wavelength of $304 \mathrm{~nm}$; whereas carvacrol dispersed in water had much weaker intensity and the wavelength of maximum intensity shifted to $309 \mathrm{~nm}$. These results indicated that the intrinsic fluorescence intensity of carvacrol was affected by its environmental polarity and an increase in polarity of environment lowered the fluorescence intensity and resulted in the red-shift of the maximum intensity wavelength. The observation agreed with the literature, as the reduced solubility in more polar water than in ethanol weakens the exposure of carvacrol for excitation by UV radiation [33].

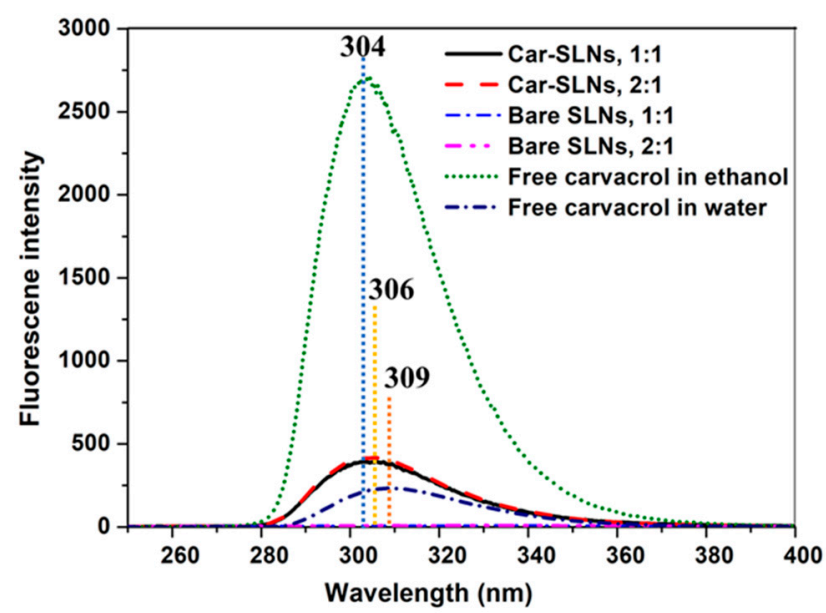

Figure 2. Fluorescence spectra of Car-SLNs with a carvacrol loading at 30\% mass of lipids and a PGMP:GMS mass ratio of 2:1 or 1:1 and the corresponding bare SLNs without carvacrol, and carvacrol pre-dissolved in ethanol and water.

The fluorescence intensity of Car-SLNs was also much lower than carvacrol dissolved in ethanol but was higher than carvacrol dispersed in water (Figure 2). Dispersion in SLNs with a small dimension (Table 2), i.e., a large surface area, likely increased the exposure than dispersing in water, but not to an extent as carvacrol molecularly dissolved in ethanol [34]. Bare SLNs without carvacrol had insignificant fluorescence, and Car-SLNs with same carvacrol loading but different PGMP:GMS mass ratios thus had similar fluorescence spectra (Figure 2). The fluorescence spectra may also provide some insights about structures of SLNs that have an overall surfactant shell and lipid core structure, and the lipid matrix can have a carvacrol core-lipid shell, lipid core-carvacrol shell, or homogeneous distribution structure [35]. If carvacrol is predominantly present in the outer shell of the lipid matrix, carvacrol will have high possibility to interact with water to cause the fluorescence quenching by water. Likewise, the predominant presence of carvacrol in the lipid matrix core may have the limited excitation by UV. The fluorescence intensity of carvacrol in SLNs being between that dissolved in ethanol and water suggests that carvacrol should be homogeneously dispersed in the SNLs, as proposed in Scheme 1.

\subsection{Release Profile of Carvacrol in SLNs}

Release kinetics was established to obtain further information about the interaction between carvacrol and other SLN components, as well as mechanisms involved in the carvacrol release. Figure 3 shows that encapsulation of carvacrol in SLNs altered the release profile, showing a slower rate initially than that of free carvacrol. For free carvacrol, 50\% release was obtained in $1.5 \mathrm{~h}$, and the osmotic equilibrium was achieved after $10 \mathrm{~h}$ with $90 \%$ release. When carvacrol was encapsulated in SLNs, $50 \%$ release of carvacrol was obtained in $6 \mathrm{~h}$, followed by a smaller rate to $80 \%$ release after $50 \mathrm{~h}$. The release profile of bioactive compounds in SLNs always followed biphasic pattern, the initial 
burst release and prolonged release [36-38]. The initial burst release results from the diffusion of carvacrol in the particle surface, and the subsequent prolonged release results from the liberation of carvacrol in the lipid matrix [39]. Therefore, the release profile of carvacrol in SLNs further verified the conclusion that carvacrol located both on the surface and core of SLNs, as previously discussed based on fluorescence spectra.

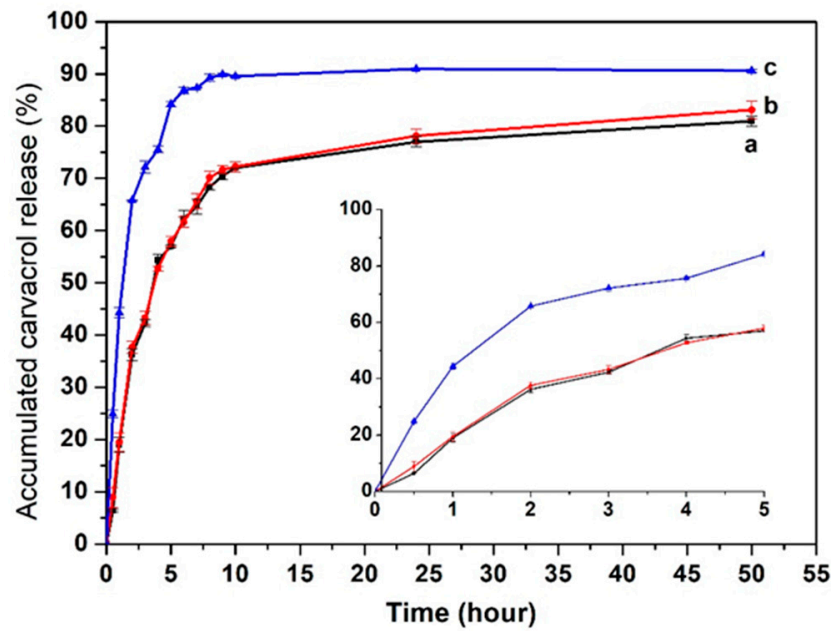

Figure 3. Release profiles of carvacrol from SLNs with a carvacrol loading at 30\% mass of lipids and a PGMP:GMS mass ratio of 2:1 (b) or 1:1 (a), with comparison to free carvacrol dissolved in ethanol (c). The inset figure shows release kinetics in the first $5 \mathrm{~h}$. The released carvacrol was detected in the release medium outside the dialysis bag. Error bars are standard derivations $(n=3)$.

\subsection{Morphology of Carvacrol-Loaded SLNs}

The detailed morphology of Car-SLNs was investigated by TEM (Figure 4). SLNs prepared with PGMP:GMS mass ratios of 2:1 and 1:1 were well dispersed with the size ranging between $100 \mathrm{~nm}$ to $200 \mathrm{~nm}$. However, Car-SLNs with a PGMP:GMS mass ratio of 1:1 were more spherical than those with a ratio of 2:1. As previously discussed, the combination of PGME and GMS with an equal molar ratio results in co-crystals with stable polymorph [40], while SLNs with polymorphic transformation from $\alpha$ - to $\beta$-form crystals can be evidenced by the formation of irregularly shaped particulates [41].

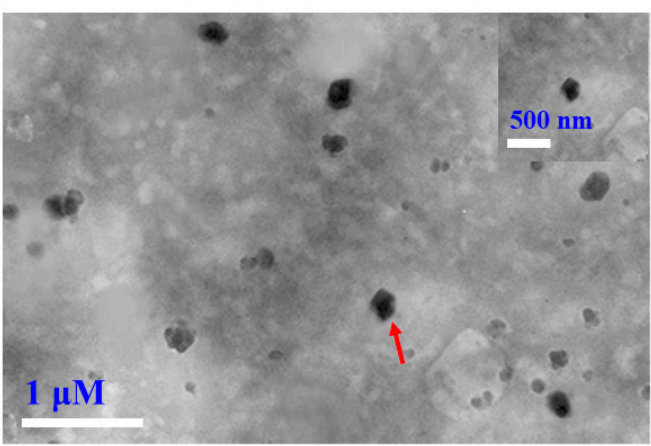

(a)

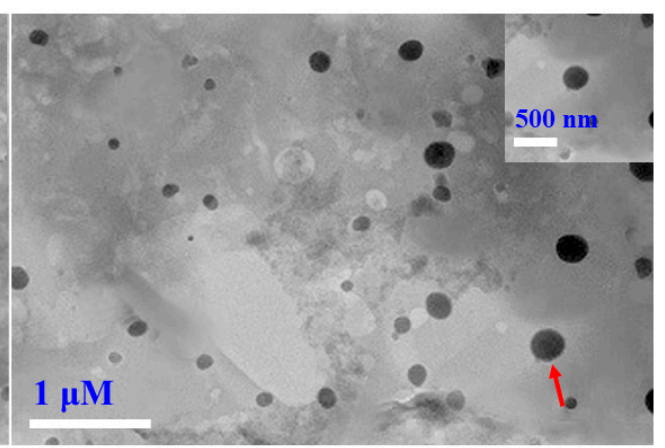

(b)

Figure 4. TEM analysis of Car-SLNs prepared with a carvacrol loading at 30\% mass of lipids and a PGMP:GMS mass ratio of 2:1 (a) or 1:1 (b). The particles shown in insert images were pointed by red arrows.

\subsection{Antimicrobial Activity}

The MIC and MBC of free and nanoencapsulated carvacrol against E. coli O157:H7 and S. aureus in comparison with the corresponding bare SLNs are summarized in Table 4. The MIC of free 
carvacrol against E. coli $\mathrm{O} 157: \mathrm{H7}$ and S. aureus was 0.250 and $0.125 \mathrm{mg} / \mathrm{mL}$, respectively, which are close to the respective reported range of $0.220-4.880$ and $0.171-0.439 \mathrm{mg} / \mathrm{mL}$ [42]. The bare SLNs also displayed antimicrobial activities against E. coli O157:H7 and S. aureus with the MIC values of 0.300 and $0.250 \mathrm{mg} / \mathrm{mL}$ at PGMP:GMS mass ratios of 2:1 and 1:1, respectively, and the higher antimicrobial activities of the latter indicated the more significant role of GMS. For the Car-SLNs, a significantly reduced MIC was found both against E. coli O157:H7 and S. aureus when compared with free carvacrol. In addition, the MBC of Car-SLNs against E. coli O157:H7 and S. aureus was investigated using sample concentration above MIC values. Car-SLNs showed the MBC values against E. coli O157:H7 and S. aureus of 0.200 and $0.125 \mathrm{mg} / \mathrm{mL}$ at the PGMP:GMS mass ratio of $2: 1$, and 0.125 and $0.100 \mathrm{mg} / \mathrm{mL}$ at the PGMP:GMS mass ratio of 1:1, respectively. These results indicated the synergistic effect of carvacrol and lipid matrix in the antimicrobial activities, with the PGMP:GMS mass ratio of 1:1 possessing more potent synergism. The synergism may be significant to lower the application level of carvacrol to overcome the negative sensory properties of carvacrol used in food preservation [43]. However, sensory studies are to be conducted in the future in specific food matrices.

Table 4. Antimicrobial activities of Car-SLNs with a carvacrol loading at 30\% mass of lipids, free carvacrol, and two bare SLNs.

\begin{tabular}{ccccc}
\hline \multirow{2}{*}{ PGMP:GMS } & \multicolumn{2}{c}{ MIC $(\mathrm{mg} / \mathrm{mL})$} & \multicolumn{2}{c}{ MBC $(\mathbf{m g} / \mathrm{mL})$} \\
\cline { 2 - 5 } & E. coli O157: H7 & S. aureus & E. coli O157: H7 & S. aureus \\
\hline 2:1, bare SLNs & 0.300 & 0.300 & 0.350 & 0.350 \\
2:1, Car-SLNs & 0.150 & 0.100 & 0.200 & 0.125 \\
1:1, bare SLNs & 0.250 & 0.250 & 0.300 & 0.300 \\
1:1, Car-SLNs & 0.125 & 0.100 & 0.125 & 0.100 \\
\hline Free carvacrol & 0.250 & 0.125 & 0.250 & 0.125 \\
\hline
\end{tabular}

The interaction of lipophilic carvacrol and phospholipid membrane of microorganisms might cause dramatic changes of the membrane structure [44], which would eventually increase passive permeability of membrane and the measured antimicrobial activity [45,46]. The MIC and MBC values of free carvacrol against E. coli O157:H7 were twice of those against S. aureus (Table 4). However, after nanoencapsulation of carvacrol in SLNs, the MIC and MBC values against E. coli O157:H7 and S. aureus became closer (Table 4), indicating that SLNs enhance the permeation of carvacrol in the outer membrane of E. coli O157:H7.

\section{Conclusions}

In conclusion, high purity PGMP was synthesized and used as a lipid matrix with GMS to fabricate the Car-SLNs. The 2:1 and 1:1 mass ratio of PGMP:GMS were feasible to prepare stable SLNs at the studied conditions and were capable of loading carvacrol at up to $30 \%$ mass of the lipids with an EE of higher than $98 \%$. Although particle growth was observed, Car-SLNs remained smaller than $200 \mathrm{~nm}$ and visually stable after 30-day storage. Fluorescence spectra indicated the carvacrol was homogeneously dispersed in the SLNs, which was further supported by the release profile. The high sphericity of Car-SLNs with a PGMP:GMS mass ratio of 1:1 suggested the absence of polymorphic transformation, which is important to SLN physical and chemical stability. The enhanced antimicrobial activities of Car-SLNs against E. coli O157:H7 and S. aureus, especially those fabricated with equal masses of PGMP and GMS show the potential applications of the studied new class of SLNs for food and cosmetic applications.

Supplementary Materials: The following are available online at http://www.mdpi.com/2079-4991/9/8/1162/s1, Figure S1: ${ }^{1} \mathrm{H}$ NMR spectra of PGMP, Figure S2: FT-IR spectra of PGMP, Figure S3: ESI-MS of PGMP. 
Author Contributions: Conceptualization, J.H. and W.Z.; methodology, J.H. and C.C.; formal analysis, S.H., J.H. and X.S.; investigation, S.H. and L.H.; writing-original draft preparation, J.H. and S.H.; assisting experimental design, data analysis and writing-review and editing, Q.Z.; supervision, W.Z.; funding acquisition, X.S.

Funding: This research was funded by Hubei Provincial Natural Science Foundation of China (No. 2018CFB356), Open Project Program of Beijing Key Laboratory of Flavor Chemistry, Beijing Technology and Business University (SPFW-2018-YB04), "One-Hundred Talents" Program of Hubei Province, and Research and Innovation Initiatives of WHPU (2018Y02). Q.Z. acknowledges hatch projects TEN00487 and 223984 supporting his time spent on this work.

Conflicts of Interest: The authors declare no conflict of interest.

\section{References}

1. Kumar, S.; Randhawa, J.K. High melting lipid based approach for drug delivery: Solid lipid nanoparticles. Mater. Sci. Eng. C 2013, 33, 1842-1852. [CrossRef] [PubMed]

2. Li, Q.; Cai, T.; Huang, Y.; Xia, X.; Cole, S.P.C.; Cai, Y. A Review of the Structure, Preparation, and Application of NLCs, PNPs, and PLNs. Nanomaterials 2017, 7, 122. [CrossRef] [PubMed]

3. Weiss, J.; Decker, E.A.; McClements, D.J.; Kristbergsson, K.; Helgason, T.; Awad, T. Solid lipid nanoparticles as delivery systems for bioactive food components. Food Biophys. 2008, 3, 146-154. [CrossRef]

4. Akhavan, S.; Assadpour, E.; Katouzian, I.; Jafari, S.M. Lipid nano scale cargos for the protection and delivery of food bioactive ingredients and nutraceuticals. Trends Food Sci. Technol. 2018, 74, 132-146. [CrossRef]

5. Aditya, N.P.; Ko, S. Solid lipid nanoparticles (SLNs): Delivery vehicles for food bioactives. RSC Adv. 2015, 5, 30902-30911. [CrossRef]

6. Müller, R.H.; Radtke, M.; Wissing, S.A. Solid lipid nanoparticles (SLN) and nanostructured lipid carriers (NLC) in cosmetic and dermatological preparations. Adv. Drug Deliv. Rev. 2002, 54, 131-155. [CrossRef]

7. Wissing, S.; Kayser, O.; Müller, R. Solid lipid nanoparticles for parenteral drug delivery. Adv. Drug Deliv. Rev. 2004, 56, 1257-1272. [CrossRef] [PubMed]

8. Shi, H.; Huang, S.; He, J.; Han, L.; Zhang, W.; Zhong, Q. 1-Laurin-3-palmitin as a novel matrix of solid lipid particles: Higher loading capacity of thymol and better Stability of dispersions than those of glyceryl monostearate and glyceryl tripalmitate. Nanomaterials 2019, 9, 489. [CrossRef] [PubMed]

9. Wang, F.C.; Marangoni, A.G. Advances in the application of food emulsifier $\alpha$-gel phases: Saturated monoglycerides, polyglycerol fatty acid esters, and their derivatives. J. Colloid Interface Sci. 2016, 483, $394-403$. [CrossRef]

10. Kuhrt, N.H.; Broxholm, R.A.; Blum, W.P. Conjoined crystals. I. composition and physical properties. J. Am. Oil Chem. Soc. 1963, 40, 725-730. [CrossRef]

11. Kuhrt, N.H.; Broxholm, R.A. Conjoined crystals. II. applications. J. Am. Oil Chem. Soc. 1963, 40, 730-733. [CrossRef]

12. Silva, F.V.; Guimares, A.G.; Silva, E.R.S.; Sousa-Neto, B.P.; Machado, F.D.F.; Quintans-Júnior, L.J.; Arcanjo, D.D.R.; Oliveira, F.A.; Oliveira, R.C.M. Anti-inflammatory and anti-ulcer activities of carvacrol, a monoterpene present in the essential oil of oregano. J. Med. Food 2012, 15, 984-991. [CrossRef] [PubMed]

13. Kumar, D.; Rawat, D.S. Synthesis and antioxidant activity of thymol and carvacrol based Schiff bases. Bioorg. Med. Chem. Lett. 2013, 23, 641-645. [CrossRef]

14. Kamimura, J.A.; Santos, E.H.; Hill, L.E.; Gomes, C.L. Antimicrobial and antioxidant activities of carvacrol microencapsulated in hydroxypropyl-beta-cyclodextrin. LWT-Food Sci. Technol. 2014, 57, 701-709. [CrossRef]

15. Veldhuizen, E.J.A.; Tjeerdsma-van Bokhoven, J.L.M.; Zweijtzer, C.; Burt, S.A.; Haagsman, H.P. Structural requirements for the antimicrobial activity of carvacrol. J. Agric. Food Chem. 2006, 54, 1874-1879. [CrossRef]

16. Liolios, C.C.; Gortzi, O.; Lalas, S.; Tsaknis, J.; Chinou, I. Liposomal incorporation of carvacrol and thymol isolated from the essential oil of Origanum dictamnus $L$. and in vitro antimicrobial activity. Food Chem. 2009, 112, 77-83. [CrossRef]

17. Khan, I.; Bahuguna, A.; Kumar, P.; Bajpai, V.K.; Kang, S.C. In vitro and in vivo antitumor potential of carvacrol nanoemulsion against human lung adenocarcinoma A549 cells via mitochondrial mediated apoptosis. Sci. Rep. 2018, 8, 144. [CrossRef]

18. Martínez-Hernández, G.B.; Amodio, M.L.; Colelli, G. Carvacrol-loaded chitosan nanoparticles maintain quality of fresh-cut carrots. Innov. Food Sci. Emerg. 2017, 41, 56-63. [CrossRef] 
19. Santos, E.H.; Kamimura, J.A.; Hill, L.E.; Gomes, C.L. Characterization of carvacrol beta-cyclodextrin inclusion complexes as delivery systems for antibacterial and antioxidant applications. LWT-Food Sci. Technol. 2015, 60, 583-592. [CrossRef]

20. Chang, Y.; McLandsborough, L.; McClements, D.J. Physicochemical properties and antimicrobial efficacy of carvacrol nanoemulsions formed by spontaneous emulsification. J. Agric. Food Chem. 2013, 61, 8906-8913. [CrossRef]

21. Wu, Y.; Luo, Y.; Wang, Q. Antioxidant and antimicrobial properties of essential oils encapsulated in zein nanoparticles prepared by liquid-liquid dispersion method. LWT-Food Sci. Technol. 2012, 48, 283-290. [CrossRef]

22. Lutton, E.S.; Stewart, C.B.; Martin, J.B. Clarification of propylene glycol monoester polymorphism. J. Am. Oil Chem. Soc. 1972, 49, 186-187. [CrossRef]

23. Raza, K.; Shareef, M.A.; Singal, P.; Sharma, G.; Negi, P.; Katare, O.P. Lipid-based capsaicin-loaded nano-colloidal biocompatible topical carriers with enhanced analgesic potential and decreased dermal irritation. J. Liposome Res. 2014, 24, 290-296. [CrossRef]

24. Shaikh, J.; Ankola, D.D.; Beniwal, V.; Singh, D.; Kumar, M.N. Nanoparticle encapsulation improves oral bioavailability of curcumin by at least 9-fold when compared to curcumin administered with piperine as absorption enhancer. Eur. J. Pharm. Sci. 2009, 37, 223-230. [CrossRef]

25. Xue, J.; Michael Davidson, P.M.; Zhong, Q. Antimicrobial activity of thyme oil co-nanoemulsified with sodium caseinate and lecithin. Int. J. Food Microbiol. 2015, 210, 1-8. [CrossRef]

26. Sjöblom, J.; Lindberg, R.; Friberg, S.E. Microemulsions-Phase equilibria characterization, structures, applications and chemical reactions. Adv. Colloid Interface Sci. 1996, 65, 125-287. [CrossRef]

27. Nayak, A.P.; Tiyaboonchai, W.; Patankar, S.; Madhusudhan, B.; Souto, E.B. Curcuminoids-loaded lipid nanoparticles: Novel approach towards malaria treatment. Colloids Surf. B 2010, 81, 263-273. [CrossRef]

28. Matsaridou, I.; Barmpalexis, P.; Salis, A.; Nikolakakis, I. The influence of surfactant HLB and oil/surfactant ratio on the formation and properties of self-emulsifying pellets and microemulsion reconstitution. AAPS PharmSciTech 2012, 13, 1319-1330. [CrossRef]

29. Taylor, P. Ostwald ripening in emulsions. Colloid Surface. A 1995, 99, 175-185. [CrossRef]

30. Chen, H.; Davidson, P.M.; Zhong, Q. Impacts of sample preparation methods on solubility and antilisterial characteristics of essential oil components in milk. Appl. Environ. Microbiol. 2014, 80, 907-916. [CrossRef]

31. Xu, R.; Wu, C.; Xu, H. Particle size and zeta potential of carbon black in liquid media. Carbon 2007, 45, 2806-2809. [CrossRef]

32. Chalier, P.; Arfa, A.B.; Preziosi-Belloy, L.; Gontard, N. Carvacrol losses from soy protein coated papers as a function of drying conditions. J. Appl. Polym. Sci. 2007, 106, 611-620. [CrossRef]

33. Noack, A.; Hause, G.; Mäder, K. Physicochemical characterization of curcuminoid-loaded solid lipid nanoparticles. Int. J. Pharm. 2012, 423, 440-451. [CrossRef]

34. Jasim, F.; Ali, F. A novel and rapid method for the spectrofluorometric determination of curcumin in curcumin spices and flavors. Microchem. J. 1992, 46, 209-214. [CrossRef]

35. Geszke-Moritz, M.; Moritz, M. Solid lipid nanoparticles as attractive drug vehicles: Composition, properties and therapeutic strategies. Mater. Sci. Eng. C 2016, 68, 982-994. [CrossRef]

36. Kuo, Y.C.; Wang, C.C. Cationic solid lipid nanoparticles with primary and quaternary amines for release of saquinavir and biocompatibility with endothelia. Colloids Surf. B 2013, 101, 101-105. [CrossRef]

37. Kakkar, V.; Singh, S.; Singla, D.; Kaur, I.P. Exploring solid lipid nanoparticles to enhance the oral bioavailability of curcumin. Mol. Nutr. Food Res. 2011, 55, 495-503. [CrossRef]

38. Lobovkina, T.; Jacobson, G.B.; Gonzalez-Gonzalez, E.; Hickerson, R.P.; Leake, D.; Kaspar, R.L.; Contag, C.H.; Zare, R.N. In vivo sustained release of siRNA from solid lipid nanoparticles. ACS Nano 2011, 5, 9977-9983. [CrossRef]

39. Muchow, M.; Maincent, P.; Müller, R.H. Lipid nanoparticles with a solid matrix (SLN $\left.{ }^{\circledR}, N_{L C}{ }^{\circledR}, \operatorname{LDC}^{\circledR}\right)$ for oral drug delivery. Drug Dev. Ind. Pharm. 2008, 34, 1394-1405. [CrossRef]

40. Sparsø, F.V. Propylene Glycol Fatty Acid Esters. In Emulsifiers in Food Technology, 2nd ed.; Norn, V., Ed.; John Wiley \& Sons, Ltd.: West Sussex, UK, 2015; pp. 231-250.

41. Arishima, T.; Sato, K. Polymorphism of POP and SOS III. Solvent crystallization of $\beta 2$ and $\beta 1$ polymorphs. J. Am. Oil Chem. Soc. 1989, 66, 1614-1617. [CrossRef] 
42. Burt, S. Essential oils: Their antibacterial properties and potential applications in foods-A review. Int. J. Food Microbiol. 2004, 94, 223-253. [CrossRef]

43. Peretto, G.; Du, W.X.; Avena-Bustillos, R.J.; Berrios Jde, J.; Sambo, P.; McHugh, T.H. Optimization of antimicrobial and physical properties of alginate coatings containing carvacrol and methyl cinnamate for strawberry application. J. Agric. Food Chem. 2014, 62, 984-990. [CrossRef]

44. Keawchaoon, L.; Yoksan, R. Preparation, characterization and in vitro release study of carvacrol-loaded chitosan nanoparticles. Colloids Surf. B 2011, 84, 163-171. [CrossRef]

45. Fett, W.F. Naturally occurring biofilms on alfalfa and other types of sprouts. J. Food Prot. 2000, 63, 625-632. [CrossRef]

46. Cristani, M.; D’Arrigo, M.; Mandalari, G.; Castelli, F.; Sarpietro, M.G.; Micieli, D.; Venuti, V.; Bisignano, G.; Saija, A.; Trombetta, D. Interaction of four monoterpenes contained in essential oils with model membranes: Implications for their antibacterial activity. J. Agric. Food Chem. 2007, 55, 6300-6308. [CrossRef]

(C) 2019 by the authors. Licensee MDPI, Basel, Switzerland. This article is an open access article distributed under the terms and conditions of the Creative Commons Attribution (CC BY) license (http://creativecommons.org/licenses/by/4.0/). 